\title{
Influence of region-of-interest designs on quantitative measurement of multimodal imaging of MR non-enhancing gliomas
}

\author{
KOJI TAKANO $^{1,2}$, MANABU KINOSHITA ${ }^{1,3}$, HIDEYUKI ARITA $^{3}$, YOSHIKO OKITA ${ }^{4}$, \\ YASUYOSHI CHIBA $^{3,5}$, NAOKI KAGAWA ${ }^{3}$, YOSHIYUKI WATANABE ${ }^{6}$, EKU SHIMOSEGAWA ${ }^{7}$, \\ JUN HATAZAWA $^{7}$, NAOYA HASHIMOTO ${ }^{8}$, YASUNORI FUJIMOTO ${ }^{3}$ and HARUHIKO KISHIMA ${ }^{3}$ \\ ${ }^{1}$ Department of Neurosurgery, Osaka International Cancer Institute, Osaka 541-8567; ${ }^{2}$ Department of \\ Neurosurgery, Toyonaka Municipal Hospital, Toyonaka, Osaka 560-8565; ${ }^{3}$ Department of Neurosurgery, \\ Osaka University Graduate School of Medicine, Suita, Osaka 565-0871; ${ }^{4}$ Department of Neurosurgery, \\ Osaka National Hospital, National Hospital Organization, Osaka 540-0006; ${ }^{5}$ Department of Neurosurgery, \\ Osaka Women's and Children's Hospital, Izumi, Osaka 594-1101; Departments of ${ }^{6}$ Radiology and \\ ${ }^{7}$ Nuclear Medicine and Tracer Kinetics, Osaka University Graduate School of Medicine, Suita, Osaka 565-0871; \\ ${ }^{8}$ Department of Neurosurgery, Kyoto Prefectural University Graduate School of Medical Science, Kyoto 602-8566, Japan
}

Received November 22, 2017; Accepted March 16, 2018

DOI: $10.3892 / \mathrm{ol} .2018 .8319$

\begin{abstract}
A number of studies have revealed the usefulness of multimodal imaging in gliomas. Although the results have been heavily affected by the method used for region of interest (ROI) design, the most discriminatory method for setting the ROI remains unclear. The aim of the present study was to determine the most suitable ROI design for ${ }^{18} \mathrm{~F}$-fluorodeoxyglucose (FDG) and ${ }^{11} \mathrm{C}$-methionine (MET) positron emission tomography (PET), apparent diffusion coefficient (ADC), and fractional anisotropy (FA) obtained by diffusion tensor imaging (DTI) from the viewpoint of grades of non-enhancing gliomas. A total of 31 consecutive patients with newly diagnosed, histologically confirmed magnetic resonance (MR) non-enhancing gliomas who underwent FDG-PET, MET-PET and DTI were retrospectively investigated. Quantitative measurements were performed using four different ROIs; hotspot/tumor center and whole tumor, constructed in either two-dimensional (2D) or three-dimensional (3D). Histopathological grading of the tumor was considered as empirical truth and the quantitative measurements obtained from each ROI was correlated with the grade of the tumor. The most discriminating ROI for non-enhancing glioma grading was different according to the different imaging modalities. 2D-hotspot/center ROI was
\end{abstract}

Correspondence to: Dr Manabu Kinoshita, Department of Neurosurgery, Osaka International Cancer Institute, 3-1-69 Otemae, Chuo-ku, Osaka 541-8567, Japan

E-mail: mail@manabukinoshita.com

Key words: glioma, magnetic resonance imaging, positron emission tomography, region of interest most discriminating for FDG-PET ( $\mathrm{P}=0.087)$, ADC map $(\mathrm{P}=0.0083)$, and FA map $(\mathrm{P}=0.25)$, whereas $3 \mathrm{D}$-whole tumor ROI was best for MET-PET $(\mathrm{P}=0.0050)$. In the majority of scenarios, 2D-ROIs performed better than 3D-ROIs. Results from the image analysis using FDG-PET, MET-PET, ADC and FA may be affected by ROI design and the most discriminating ROI for non-enhancing glioma grading was different according to the imaging modality.

\section{Introduction}

Advanced magnetic resonance imaging (MRI) methods, such as diffusion tensor imaging (DTI) and perfusion weighted imaging, as well as positron emission tomography (PET) are useful for clinical management of brain tumors. Many studies have revealed their usefulness for diagnosis, grading, prognosis, delineation, and assessment of genetic mutations, especially in gliomas (1-10). Although they are promising modalities, their usefulness can still be improved. For example, Lee et al reported that high-grade gliomas have lower apparent diffusion coefficient (ADC) than low-grade gliomas (3), but Stadlbauer et al reported otherwise (6). In contrast, Liu et al found no significant difference between low- and high-grade gliomas (8).

Whenever we assess brain tumors with these modalities, the region of interest (ROI) must first be designed. Although results are heavily affected by the method used for ROI design, the most discriminant method to set the ROI remains unclear. Some researchers set the ROI on the hotspot or on the center of the tumor, whereas others set it on the whole gadolinium-enhanced/fluid attenuation inversion recovery (FLAIR)-high lesion or on the margin within the tumor. Similarly, some use two-dimensional (2D)-ROI, whereas others use three-dimensional (3D)-ROI. These various methods for ROI design hamper direct comparison of the 
results between different studies, resulting in controversy. For example, Liu et al found higher fractional anisotropy (FA) in high-grade gliomas than in low-grade gliomas with the ROI set on the hotspot (8), whereas Goebell et al reported otherwise, with the ROI set on the margin of the tumor (1). Therefore, a standardized and discriminant method for ROI design is required for both clinical and research purposes, and may resolve the contradictions of past reports.

The purpose of our study was to elucidate the impact of ROI design in various imaging modalities on the obtained image analysis results. In the present study, we assessed four types of ROIs (hotspot/tumor center or whole tumor, 2D or 3D) in ${ }^{18} \mathrm{~F}$-fluorodeoxyglucose (FDG)-PET, ${ }^{11} \mathrm{C}$-methionine (MET)-PET, ADC, and FA obtained by DTI, from the viewpoint of the grade of non-enhancing gliomas in which ROI setting is more difficult than in enhancing gliomas.

\section{Materials and methods}

Patients and data collection. We collected data from patients with newly diagnosed non-enhancing supratentorial gliomas that were histologically confirmed who underwent MRI-DTI, FDG-PET, and MET-PET for pre-surgical examination (17 men, 14 women; mean age, $40.5 \pm 16.0$ years; World Health Organization grade II, n=21; grade III, n=10). Four of the 21 grade II gliomas and two of the 10 grade III gliomas showed oligodendrocytic subtypes. Histological grading was performed according to the World Health Organization 2007 criteria after partial $(<95 \%$ tumor removal) or total ( $\geq 95 \%$ tumor removal) resection of the lesion or stereotactic biopsy (total resection, $\mathrm{n}=10$; partial resection, $\mathrm{n}=18$; stereotactic biopsy, $n=3$ ). Detailed characteristics of patients are given in Table I. Use of clinical data for research purposes was approved by the local institutional review board. All procedures performed in studies involving human participants were in accordance with the ethical standards of the institutional and/or national research committee and with the 1964 Helsinki declaration and its later amendments or comparable ethical standards. Written informed consent was previously obtained for using radiological data for future clinical research from all individual participants included in the study.

PET methods. PET studies were performed with an Eminence-G (Shimadzu, Kyoto, Japan). Synthesis of MET was performed according to the method described by Berger et al (11), and MET was injected intravenously at a dose of $3 \mathrm{MBq} / \mathrm{kg}$. Tracer accumulation was recorded for 12 min in 59 or 99 transaxial sections over the entire brain. Summed activity from 20 to $32 \mathrm{~min}$ after tracer injection was used for image reconstruction. For FDG-PET, after a 10-min transmission scan, FDG was injected intravenously at a dose of 3.7 MBq/kg. Tracer accumulation was recorded in 3D mode for 12 min in 59 or 99 transaxial sections from the entire brain. Total activity from 45 to $57 \mathrm{~min}$ after tracer injection was used for image reconstruction. Both images were stored in 256x256x59 or 99 anisotropic voxels, with each voxel being 1x1x2.6 mm.

MRI. All patients were studied using a 3.0-T MRI scanner (Signa HDxt or DiscoveryMR 750; GE Medical Systems, Milwaukee, WI, USA) within a week before the operation.
T1-weighted imaging with gadolinium enhancement was used to select patients with non-enhancing gliomas. T2-weighted (T2) or FLAIR images were acquired in all cases for delineation of tumors. DTI was acquired using a single-shot echo-planar imaging technique with $\mathrm{TE}=80$ and $\mathrm{TR}=10,000$. Diffusion gradient encoding in 25 directions with $b=2,000 \mathrm{~s} / \mathrm{mm}^{2}$ and an additional measurement without the diffusion gradient $\left(b=0 \mathrm{~s} / \mathrm{mm}^{2}\right.$ ) were performed (12). A parallel imaging technique was used to record data with a $128 \times 128$ spatial resolution for a $260 \times 260 \mathrm{~mm}$ field of view. A total of 50 sections were obtained, with a section thickness of $3 \mathrm{~mm}$ and no intersection gap. ADC and FA maps were processed using Diffusion Toolkit (Martinos Center for Biomedical Imaging, Massachusetts General Hospital, Charlestown, MA, USA; http://www.trackvis.org/dtk/).

Image fusion. After all images were obtained, PET images, $\mathrm{ADC}$, and FA maps were all registered to T2 or FLAIR images using VINCI image analysis software (Max-Planck Institute for Neurological Research, Cologne, Germany; http://www. nf.mpg.de/vinci/). Correct co-registration of images was visually confirmed. After image registration was complete, all image sets were converted to anisotropic images (256x256x59 or $99,1 \times 1 \times 2.6 \mathrm{~mm}$ ), enabling further analysis. For PET images, standardized uptake value (SUV) of the contralateral tumor-unaffected gray matter in the axial plane at the level of the thalamus was averaged, and the derived value was used to normalize SUV in a voxel-wise manner, enabling reconstruction of tumor-to-normal tissue $(\mathrm{T} / \mathrm{N})$ ratio images. All datasets were exported to in-house software written in MATLAB 7.14 (MathWorks, Natick, MA, USA) for further analysis.

ROI design and analysis. We designed four types of ROIs: 2D-whole tumor (2D-WT), 3D-whole tumor (3D-WT), 2D-hotspot/center (2D-HC), and 3D-hotspot/center (3D-HC) in four imaging maps: FDG-PET, MET-PET, ADC, and FA. To design 2D-ROIs, an axial plane through the hotspot was visually selected. If no hotspot was found, an axial plane through the center of the tumor was used. To design 3D-HC ROIs, three or more consecutive planes through the hotspot or the center of the tumor were selected. To design WT ROIs, the T2/FLAIR high lesion was delineated manually (Fig. 1). To design HC ROIs, the hotspot was delineated manually, taking care to not contaminate the hotspot with tumor margins, necrosis, large vessels, ventricles, and sulci (Fig. 1), i.e., hotspots were visually identified in FDG-PET, MET-PET, ADC and FA, followed by manual segmentation of that region. If no hotspot was found, the center of the T2/FLAIR high lesion that was at least $5 \mathrm{~mm}$ from the tumor margin was delineated manually, taking care to not contaminate the ROI with the above-mentioned structures (Fig. 1). All ROIs were designed by the leading author, who has great experience in surgical neuro-oncology, supervised by the corresponding author.

Maximum $\mathrm{T} / \mathrm{N}$ values $\left(\mathrm{T} / \mathrm{N}_{\max }\right)$ of both FDG-PET and MET-PET, minimum ADC $\left(\mathrm{ADC}_{\text {min }}\right)$, and maximum FA $\left(\mathrm{FA}_{\max }\right)$ within each ROI were calculated. All values are reported as the mean \pm standard deviation.

Statistical analysis. Statistical analysis was performed with JMP version 12 software (SAS Institute, Inc., Cary, NC, 
Table I. Patient characteristics.

\begin{tabular}{|c|c|c|c|c|c|c|}
\hline Patient no. & $\begin{array}{c}\text { Age at surgery } \\
\text { (years) }\end{array}$ & Gender & $\begin{array}{c}\text { Histological } \\
\text { diagnosis }\end{array}$ & $\begin{array}{l}\text { Tumor } \\
\text { grade }\end{array}$ & Tumor location & $\begin{array}{l}\text { Type of } \\
\text { surgery }\end{array}$ \\
\hline 1 & 11 & $\mathrm{M}$ & DA & II & L frontal & TR \\
\hline 2 & 25 & $\mathrm{M}$ & DA & II & L temporal & PR \\
\hline 3 & 25 & $\mathrm{~F}$ & DA & II & L insula & PR \\
\hline 4 & 25 & M & DA & II & L temporal & PR \\
\hline 5 & 26 & M & DA & II & $\mathrm{R}$ frontal & PR \\
\hline 6 & 28 & $\mathrm{M}$ & DA & II & L temporal & PR \\
\hline 7 & 30 & $\mathrm{~F}$ & DA & II & L temporal & PR \\
\hline 8 & 31 & M & DA & II & $\mathrm{R}$ insula & TR \\
\hline 9 & 41 & M & DA & II & L temporal & TR \\
\hline 10 & 41 & $\mathrm{~F}$ & DA & II & L frontal & TR \\
\hline 11 & 42 & F & DA & II & L frontal & TR \\
\hline 12 & 43 & $\mathrm{~F}$ & DA & II & L insula & TR \\
\hline 13 & 43 & $\mathrm{M}$ & DA & II & $\mathrm{R}$ temporal & PR \\
\hline 14 & 50 & $\mathrm{~F}$ & DA & II & L thalamus & B \\
\hline 15 & 59 & $\mathrm{~F}$ & DA & II & L frontal & PR \\
\hline 16 & 65 & $\mathrm{~F}$ & DA & II & $\mathrm{R}$ insula & PR \\
\hline 17 & 67 & $\mathrm{M}$ & DA & II & L insula-temporal & PR \\
\hline 18 & 33 & M & OA & II & L frontal & PR \\
\hline 19 & 36 & $\mathrm{M}$ & OA & II & $\mathrm{R}$ frontal & TR \\
\hline 20 & 35 & M & $\mathrm{O}$ & II & $\mathrm{R}$ basal ganglia & PR \\
\hline 21 & 40 & M & $\mathrm{O}$ & II & $\mathrm{R}$ frontal & TR \\
\hline 22 & 19 & $\mathrm{~F}$ & AA & III & L thalamus & B \\
\hline 23 & 28 & $\mathrm{~F}$ & AA & III & L frontal & TR \\
\hline 24 & 29 & M & AA & III & $\mathrm{R}$ thalamus & PR \\
\hline 25 & 31 & $\mathrm{~F}$ & AA & III & Bilateral frontal & PR \\
\hline 26 & 48 & $\mathrm{M}$ & AA & III & L insula-talamus & PR \\
\hline 27 & 62 & $\mathrm{~F}$ & AA & III & L temporal & PR \\
\hline 28 & 67 & $\mathrm{~F}$ & AA & III & L thalamus & B \\
\hline 29 & 76 & M & AA & III & L parietal & PR \\
\hline 30 & 39 & $\mathrm{~F}$ & $\mathrm{AOA}$ & III & $\begin{array}{l}\mathrm{R} \text { frontal- } \\
\text { insula-temporal }\end{array}$ & PR \\
\hline 31 & 59 & M & AOA & III & $\mathrm{R}$ frontal & TR \\
\hline
\end{tabular}

M, male; F, female; DA, defuse astrocytoma; OA, oligoastrocytoma; O, oligodendroglioma; AA, anaplastic astrocytoma; AOA, anaplastic oligoastrocytoma; L, left; R, right; TR, total resection (>95\%); PR, partial resection; B, biopsy; WHO, World Health Organisation.

USA). A threshold level of 0.05 was established for statistical significance. The Mann-Whitney U test was used for group comparisons.

\section{Results}

Difference in $T / N_{\max }$ of FDG-PET between grade II and III non-enhancing gliomas was the greatest for the $2 D$-hotspot/center ROI. The $\mathrm{T} / \mathrm{N}_{\max }$ of FDG-PET for grade II and III gliomas were $1.02 \pm 0.36$ and $1.02 \pm 0.15$ by $2 \mathrm{D}-\mathrm{WT}$ ROI, $1.14 \pm 0.34$ and $1.15 \pm 0.15$ by 3D-WT ROI, $0.76 \pm 0.40$ and $0.86 \pm 0.21$ by $2 \mathrm{D}-\mathrm{HC}$ ROI, and $0.85 \pm 0.39$ and $0.88 \pm 0.20$ by 3D-HC ROI, respectively (Table II, FDG section). Although these differences were not significant $(\mathrm{P}=0.41,0.85,0.087$, and 0.28 , respectively), the difference between grade II and III gliomas tended to increase when utilizing 2D-HC ROI. T/ $\mathrm{N}_{\max }$ by both $2 \mathrm{D}-\mathrm{WT}$ and $3 \mathrm{D}-\mathrm{WT}$ ROIs and $2 \mathrm{D}-\mathrm{HC}$ and $3 \mathrm{D}-\mathrm{HC}$ ROIs showed strong positive correlations $\left(\mathrm{R}^{2}=0.83\right.$ and 0.95 , respectively). On the other hand, $\mathrm{T} / \mathrm{N}_{\max }$ by $2 \mathrm{D}-\mathrm{WT}$ and $2 \mathrm{D}-\mathrm{HC}$ ROIs or 3D-WT and 3D-HC ROIs only showed moderate correlations $\left(\mathrm{R}^{2}=0.77\right.$ and 0.77 , respectively) (Table III).

3D-whole tumor ROI showed the best performance for discriminating grade II and III non-enhancing gliomas for MET-PET. The $\mathrm{T} / \mathrm{N}_{\max }$ of MET-PET for grade II and III gliomas were $1.90 \pm 0.66$ and $2.63 \pm 0.51$ by $2 \mathrm{D}-\mathrm{WT}$ ROI, $1.92 \pm 0.64$ and $2.67 \pm 0.48$ by $3 \mathrm{D}-\mathrm{WT}$ ROI, $1.71 \pm 0.61$ and $2.30 \pm 0.77$ by $2 \mathrm{D}-\mathrm{HC}$ ROI, and $1.79 \pm 0.60$ and $2.37 \pm 0.72$ by 3D-HC ROI, respectively (Table II, MET section). Although all these differences were statistically significant $(\mathrm{P}=0.0056$, 
Whole tumor ROI

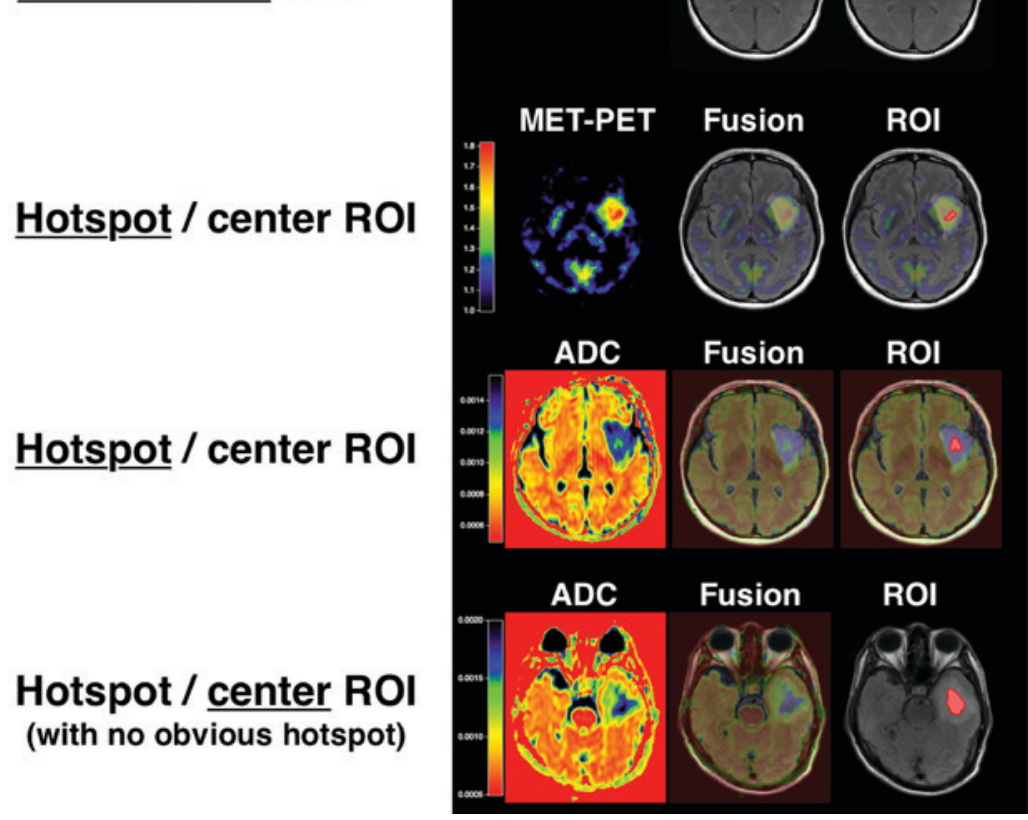

Figure 1. Presentation of ROI construction. Examples of whole tumor ROI (first row) and hotspot/center ROIs (second row, MET-PET; third row, ADC) in the same patient are illustrated. Hotspot/center ROIs are often not identical among different imaging modalities. The fourth row is an example of ROI construction in which no hotspot was observed. In these cases the center of the T2/FLAIR high lesion was delineated manually taking care not to contaminate the ROI with necrosis, large vessels, ventricles and sulci in an axial plane through the center of the T2/FLAIR high lesion. To ensure avoidance of these types of contamination, the ROI was delineated at least $5 \mathrm{~mm}$ away from the tumor margin. ROI, region of interest; ADC, apparent diffusion coefficient; MET, ${ }^{11} \mathrm{C}-\mathrm{methionine}$; PET, positron emission tomography; FLAIR, fluid attenuation inversion recovery.

$0.0050,0.049$, and 0.040 , respectively), the difference between grade II and III gliomas tended to increase when utilizing 3D-WT ROIs. The area under the curve (AUC) of the receiver operating characteristic (ROC) curve was largest for 3D-WT ROI for discriminating grade II and III non-enhancing gliomas (Fig. 2A, AUC $=0.82$ ). $\mathrm{T} / \mathrm{N}_{\max }$ by both $2 \mathrm{D}-\mathrm{WT}$ and 3D-WT ROIs and 2D-HC and 3D-HC ROIs showed strong positive correlations $\left(\mathrm{R}^{2}=0.98\right.$ and 0.97 , respectively). On the other hand, $\mathrm{T} / \mathrm{N}_{\max }$ by $2 \mathrm{D}-\mathrm{WT}$ and $2 \mathrm{D}-\mathrm{HC}$ ROIs or $3 \mathrm{D}-\mathrm{WT}$ and 3D-HC ROIs only showed moderate correlations $\left(\mathrm{R}^{2}=0.77\right.$ and 0.78 , respectively) (Table III).

$2 D$-hotspot/center ROI showed the best performance for discriminating grade II and III non-enhancing gliomas for $A D C . \mathrm{ADC}_{\min }$ for grade II and III gliomas were $0.66 \pm 0.18$ and $0.54 \pm 0.14$ by $2 \mathrm{D}-\mathrm{WT}$ ROI, $0.39 \pm 0.28$ and $0.40 \pm 0.14$ by 3D-WT ROI, $1.08 \pm 0.33$ and $0.73 \pm 0.29$ by $2 \mathrm{D}-\mathrm{HC}$ ROI, and $0.91 \pm 0.25$ and $0.57 \pm 0.27$ by $3 \mathrm{D}-\mathrm{HC}$ ROI, respectively (Table II, ADC section). All these differences except 3D-WT ROI were statistically significant $(\mathrm{P}=0.024,0.63,0.0083$, and 0.027 , respectively). The difference between grade II and III gliomas was greatest when applying 2D-HC ROI. The AUC of the ROC curve was also greatest for 2D-HC ROI for discriminating grade II and III non-enhancing gliomas (Fig. 2B, AUC $=0.80$ ). $\mathrm{ADC}_{\min }$ by both $2 \mathrm{D}-\mathrm{WT}$ and $3 \mathrm{D}-\mathrm{WT}$ ROIs and $2 \mathrm{D}-\mathrm{HC}$ and 3D-HC ROIs showed strong positive correlations $\left(\mathrm{R}^{2}=0.98\right.$ and 0.97 , respectively). Although $\mathrm{ADC}_{\min }$ by $2 \mathrm{D}-\mathrm{HC}$ ROI and $3 \mathrm{D}$-HC ROI showed a moderate correlation $\left(\mathrm{R}^{2}=0.75\right), \mathrm{ADC}_{\min }$ by $2 \mathrm{D}-\mathrm{WT}$ ROI and 3D-WT ROI did not show this trend
$\left(\mathrm{R}^{2}=0.12\right)$. Similarly, $\mathrm{ADC}_{\min }$ by $2 \mathrm{D}-\mathrm{WT}$ and $2 \mathrm{D}-\mathrm{HC}$ ROIs or 3D-WT and 3D-HC ROIs did not show significant correlations $\left(\mathrm{R}^{2}=0.47\right.$ and 0.11 , respectively) (Table III).

Difference in FA between grade II and III non-enhancing gliomas was greatest for $2 D$-hotspot/center ROI. The $\mathrm{FA}_{\max }$ for grade II and III gliomas were $0.51 \pm 0.17$ and $0.51 \pm 0.15$ by $2 \mathrm{D}-\mathrm{WT}$ ROI, $0.68 \pm 0.15$ and $0.68 \pm 0.14$ by $3 \mathrm{D}-\mathrm{WT}$ ROI, $0.30 \pm 0.11$ and $0.38 \pm 0.17$ by $2 \mathrm{D}-\mathrm{HC}$ ROI, and $0.39 \pm 0.12$ and $0.44 \pm 0.19$ by $3 \mathrm{D}-\mathrm{HC}$ ROI, respectively (Table II, FA section). Although these differences were not significant $(\mathrm{P}=0.95$, $0.92,0.25$, and 0.66 , respectively), the difference between grade II and III gliomas tended to increase when applying 2D-HC ROI. FA $\max _{\max }$ by 2D-WT and 3D-WT ROIs did not show a strong correlation, whereas $2 \mathrm{D}-\mathrm{HC}$ and $3 \mathrm{D}-\mathrm{HC}$ ROIs showed moderate positive correlations $\left(\mathrm{R}^{2}=0.40\right.$ and 0.64 , respectively). $\mathrm{FA}_{\max }$ by $2 \mathrm{D}-\mathrm{WT}$ and $2 \mathrm{D}-\mathrm{HC}$ ROIs or $3 \mathrm{D}-\mathrm{WT}$ and 3D-HC ROIs showed little correlation $\left(\mathrm{R}^{2}=0.21\right.$ and 0.24 , respectively) (Table III).

\section{Discussion}

The present study aimed to identify the best ROI that should be used for analyzing various imaging modalities for discriminating non-enhancing gliomas. The most discriminating ROI for non-enhancing glioma grading was different according to different imaging modalities. 2D-hotspot/center ROI was most discriminating for FDG-PET, ADC map, and FA map, whereas 3D-whole tumor ROI was best for MET-PET. 
Table II. T/ $\mathrm{N}_{\max }$ of various imaging modalities using different ROIs in grade II and III gliomas.

\section{A, FDG-PET}

\begin{tabular}{|c|c|c|c|c|}
\hline ROI & Dimension & Grade II & Grade III & P-value \\
\hline \multirow[t]{2}{*}{ Whole tumor } & $2 \mathrm{D}$ & $1.02 \pm 0.36$ & $1.02 \pm 0.15$ & 0.4099 \\
\hline & $3 \mathrm{D}$ & $1.14 \pm 0.34$ & $1.15 \pm 0.15$ & 0.8492 \\
\hline \multirow[t]{2}{*}{ Hotspot/center } & $2 \mathrm{D}$ & $0.76 \pm 0.40$ & $0.86 \pm 0.21$ & 0.0870 \\
\hline & $3 \mathrm{D}$ & $0.85 \pm 0.39$ & $0.88 \pm 0.20$ & 0.2812 \\
\hline
\end{tabular}

\section{B, MET-PET}

\begin{tabular}{lcccr}
\hline ROI & Dimension & Grade II & Grade III & P-value \\
\hline Whole tumor & 2D & $1.90 \pm 0.66$ & $2.63 \pm 0.51$ & 0.0056 \\
& 3D & $1.92 \pm 0.64$ & $2.67 \pm 0.48$ & 0.0050 \\
Hotspot/center & 2D & $1.71 \pm 0.61$ & $2.30 \pm 0.77$ & 0.0494 \\
& 3D & $1.79 \pm 0.60$ & $2.37 \pm 0.72$ & 0.0404 \\
\hline
\end{tabular}

$\mathrm{C}, \operatorname{ADC}\left(\times 10^{-3} \mathrm{~mm}^{2} / \mathrm{s}\right)$

\begin{tabular}{lcccc}
\hline ROI & Dimension & Grade II & Grade III & P-value \\
\hline Whole tumor & 2D & $0.66 \pm 0.18$ & $0.54 \pm 0.14$ & 0.0238 \\
& 3D & $0.39 \pm 0.28$ & $0.40 \pm 0.14$ & 0.6270 \\
Hotspot/center & 2D & $1.08 \pm 0.33$ & $0.73 \pm 0.29$ & 0.0083 \\
& 3D & $0.91 \pm 0.25$ & $0.57 \pm 0.27$ & 0.0265
\end{tabular}

D, FA

\begin{tabular}{lcccc}
\hline ROI & Dimension & Grade II & Grade III \\
\hline Whole tumor & 2D & $0.51 \pm 0.17$ & $0.51 \pm 0.15$ & P-value \\
& 3D & $0.68 \pm 0.15$ & $0.68 \pm 0.14$ & 0.9495 \\
Hotspot/center & 2D & $0.30 \pm 0.11$ & $0.38 \pm 0.17$ & 0.2452 \\
& 3D & $0.39 \pm 0.12$ & $0.44 \pm 0.19$ & 0.6573
\end{tabular}

$\mathrm{T} / \mathrm{N}$, tumor-to-normal; ROI, region of interest; FDG, ${ }^{18} \mathrm{~F}$-fluorodeoxyglucose; MET, ${ }^{11} \mathrm{C}$-methionine; ADC, apparent diffusion coefficient; FA, fractional anisotropy.

Table III. Correlation coefficient for each combination of ROIs.

$\mathrm{R} 2$

\begin{tabular}{lllll}
\cline { 3 - 5 } ROI & FDG & MET & ADC & FA \\
\hline 2D-whole tumor vs. 3D-whole tumor & 0.83 & 0.98 & 0.12 & 0.40 \\
2D-hotspot/center vs. 3D-hotspot/center & 0.95 & 0.97 & 0.75 & 0.64 \\
2D-whole tumor vs. 2D-hotspot/center & 0.77 & 0.77 & 0.47 & 0.21 \\
3D-whole tumor vs. 3D-hotspot/center & 0.77 & 0.78 & 0.11 & 0.24
\end{tabular}

ROI, region of interest; FDG, ${ }^{18} \mathrm{~F}$-fluorodeoxyglucose; MET, ${ }^{11} \mathrm{C}$-methionine; ADC, apparent diffusion coefficient; FA, fractional anisotropy.

FDG uptake is thought to correlate with glucose metabolism within tissues, and high uptake in gliomas has been reported to reflect malignancy $(5,9,13)$. However, the brain has highly active glucose metabolism, especially the gray matter. 

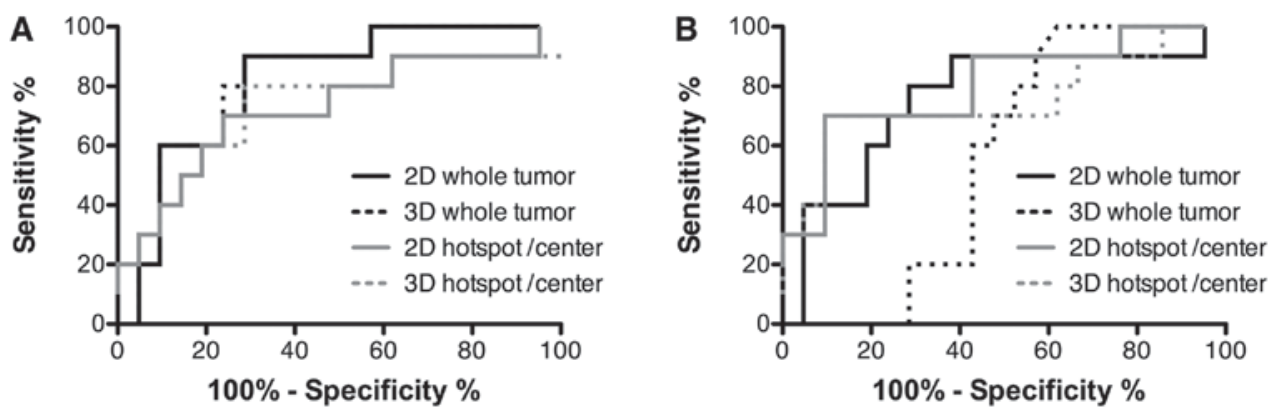

Figure 2. Receiver operating characteristic curves for differentiating grade II and III non-enhancing gliomas based on different regions of interest are presented. Image analysis for ${ }^{11} \mathrm{C}$-methionine-positron emission tomography (A) and the apparent diffusion coefficient map (B) are shown.

On the other hand, FDG uptake of lower grade gliomas is usually in between that of gray and white matter. As gliomas are poorly delineated tumors containing normal tissues at the tumor margins, the tumor margin adjacent to the gray matter could have higher FDG uptake than one would expect based on only the malignancy. This contamination could result in poor discrimination using whole tumor ROIs. In addition, FDG-PET is more useful for high-grade than low-grade gliomas because high-grade gliomas tend to have higher FDG uptake than the gray matter (9). Supported by these reports, the differences in FDG uptake between grade II and III gliomas were not statistically significant no matter what type of ROIs were used for analysis, as the present cohort was comprised of only grade II and grade III gliomas.

The result for ADC and FA also highlights the importance of excluding normal tissue contamination into the ROIs. ADC is negatively correlated with cell density within a tissue. As higher cell density indicates higher malignancy, lower ADC is thought to be the hallmark of higher-grade gliomas $(3,4,14)$. However, the normal white matter has the same or even a lower level of ADC than malignant gliomas, and the tumor margin adjacent to the white matter could show lower ADC than one would expect based on only the cell density. Our result for ADC is consistent with the past report by Fan et al, which showed lower ADC values in the solid portions of anaplastic gliomas, but not in peritumoral regions (15). Although FA is also positively correlated with cell density, it can also be affected by intact neural fibers within the tumor and around the tumor margins, with a much higher FA level than malignant gliomas $(7,8,16,17)$. Thus, use of FA for glioma grading is difficult. In the present study, ADC and FA tended to differ greatly depending on the ROI design rather than FDG and MET.

In contrast to FDG-PET, ADC, and FA, whole tumor ROIs were better than hotspot/center ROIs for MET-PET. MET uptake is thought to correlate with amino acid metabolism within a tissue, and higher uptake is indicative of higher-grade gliomas (9,18-20). As gliomas show higher MET uptake than normal gray matter, MET-PET is free from normal tissue tracer uptake contamination $(21,22)$. The cause for hotspot/center ROIs being inferior to whole tumor ROIs for tumor grading can be explained by technical difficulty in visually identifying the highest tracer uptake location. In addition, whole tumor ROIs are easy to design with little inter-observer difference, whereas designing hotspot/center ROIs requires experience with finding hotspots and avoiding cysts, necrosis, or normal tissue, which components could contaminate the measurements that should be done within the tumor.

As gliomas expand three-dimensionally, 3D-ROIs were at first expected to be superior to 2D-ROIs. Contrary to our expectation, 2D-ROIs performed as well as 3D-ROIs. This finding is similar to previous reports focusing on response assessment using different ROIs. In RECIST (Response Evaluation Criteria in Solid Tumors), tumor size is measured one-dimensionally because of its convenience and accuracy. Several studies have compared the RECIST criteria with two-dimensional, three-dimensional, and volumetric measurements in high-grade gliomas and found that they were all in good concordance with each other (23-25). The findings of our present study may indicate that two-dimensional assessment is sufficient not only for size assessment but also for functional assessment such as grading and prognosis. 2D-ROIs could be preferable especially for FDG, $\mathrm{ADC}$, and FA.

Limitations of our study should be noted. In the present study, we evaluated the superiority of ROI design methods considering histopathological grading of non-enhancing gliomas as a ground truth. Whether our result is valid for other purposes such as prognosis or assessment of genetic mutations is unclear. It is also of note that this investigation heavily depends on the operator(s) that designed the ROIs.

In conclusion, results from image analysis using FDG-PET, MET-PET, ADC, and FA could be affected by ROI design, and the most discriminating ROI for non-enhancing glioma grading was different according to imaging modality. ROI for FDG-PET, ADC, and FA should be narrowed down to the hotspot or the center of the tumor, whereas ROI for MET-PET should include the whole tumor. Two-dimensional ROI may be sufficient for grading. Attention should be paid to the ROI designs used for interpreting results using the abovementioned imaging modalities.

\section{Acknowledgements}

Not applicable.

\section{Funding}

This study was supported by JSPS KAKENHI (grant nos. 16K20008, 16K10778 and 17H05308), Osaka Medical Research Foundation for Intractable Diseases and Uehara Memorial Foundation. 


\section{Availability of data and materials}

The datasets used and/or analyzed during the current study are available from the corresponding author on reasonable request.

\section{Authors' contributions}

KT, MK, HA, YO, YC, NK, YW, ES, JH, NH, YF AND HK all obtained clinical data. KT designed each of the ROIs, and analyzed the images and data. MK supervised the whole study. All authors read and approved the final manuscript.

\section{Ethics approval and consent to participate}

Use of clinical data for research purposes was approved by the local institutional review board. All procedures performed in studies involving human participants were in accordance with the ethical standards of the institutional and/or national research committee and with the 1964 Helsinki declaration and its later amendments or comparable ethical standards. Written informed consent was obtained from all individual participants included in the study.

\section{Consent for publication}

Not applicable.

\section{Competing interests}

The authors declare that they have no competing interests.

\section{References}

1. Goebell E, Paustenbach S, Vaeterlein O, Ding XQ, Heese O, Fiehler J, Kucinski T, Hagel C, Westphal M and Zeumer H: Low-grade and anaplastic gliomas: Differences in architecture evaluated with diffusion-tensor MR imaging. Radiology 239: 217-222, 2006.

2. Lee EJ, Lee SK, Agid R, Bae JM, Keller A and Terbrugge K: Preoperative grading of presumptive low-grade astrocytomas on MR imaging: Diagnostic value of minimum apparent diffusion coefficient. AJNR Am J Neuroradiol 29: 1872-1877, 2008.

3. Lee HY, Na DG, Song IC, Lee DH, Seo HS, Kim JH and Chang KH: Diffusion-tensor imaging for glioma grading at 3-T magnetic resonance imaging: Analysis of fractional anisotropy and mean diffusivity. J Comput Assist Tomogr 32: 298-303, 2008

4. Higano S, Yun X, Kumabe T, Watanabe M, Mugikura S, Umetsu A, Sato A, Yamada T and Takahashi S: Malignant astrocytic tumors: Clinical importance of apparent diffusion coefficient in prediction of grade and prognosis. Radiology 241: 839-846, 2006.

5. Van Laere K, Ceyssens S, Van Calenbergh F, de Groot T, Menten J, Flamen P, Bormans G and Mortelmans L: Direct comparison of 18F-FDG and 11C-methionine PET in suspected recurrence of glioma: Sensitivity, inter-observer variability and prognostic value. Eur J Nucl Med Mol Imaging 32: 39-51, 2005.

6. Stadlbauer A, Ganslandt O, Buslei R, Hammen T, Gruber S, Moser E, Buchfelder M, Salomonowitz E and Nimsky C: Gliomas: Histopathologic evaluation of changes in directionality and magnitude of water diffusion at diffusion-tensor MR imaging. Radiology 240: 803-810, 2006.

7. Kinoshita M, Hashimoto N, Goto T, Kagawa N, Kishima H, Izumoto S, Tanaka H, Fujita $\mathrm{N}$ and Yoshimine T: Fractional anisotropy and tumor cell density of the tumor core show positive correlation in diffusion tensor magnetic resonance imaging of malignant brain tumors. Neuro Image 43: 29-35, 2008.

8. Liu X, Tian W, Kolar B, Yeaney GA, Qiu X, Johnson MD and Ekholm S: MR diffusion tensor and perfusion-weighted imaging in preoperative grading of supratentorial nonenhancing gliomas. Neuro Oncol 13: 447-455, 2011.
9. Singhal T, Narayanan TK, Jacobs MP, Bal C and Mantil JC: 11C-methionine PET for grading and prognostication in gliomas: A comparison study with 18F-FDG PET and contrast enhancement on MRI. J Nucl Med 53: 1709-1715, 2012.

10. Okita Y, Nonaka M, Shofuda T, Kanematsu D, Yoshioka E, Kodama Y, Mano M, Nakajima S and Kanemura Y: (11) C-methinine uptake correlates with MGMT promoter methylation in nonenhancing gliomas. Clin Neurol Neurosurg 125: 212-216, 2014.

11. Berger G, Maziere M, Knipper R, Prenant C and Comar D: Automated synthesis of $11 \mathrm{C}$-labelled radiopharmaceuticals: Imipramine, chlorpromazine, nicotine and methionine. Int $\mathrm{J}$ Appl Radiat Isot 30: 393-399, 1979.

12. Yoshiura T, Mihara F, Tanaka A, Ogomori K, Ohyagi Y, Taniwaki T, Yamada T, Yamasaki T, Ichimiya A, Kinukawa N, et al: High b value diffusion-weighted imaging is more sensitive to white matter degeneration in Alzheimer's disease. NeuroImage 20: 413-419, 2003.

13. Di Chiro G, DeLaPaz RL, Brooks RA, Sokoloff L, Kornblith PL, Smith BH, Patronas NJ, Kufta CV, Kessler RM, Johnston GS, et al: Glucose utilization of cerebral gliomas measured by [18F] fluorodeoxyglucose and positron emission tomography. Neurology 32: 1323-1329, 1982

14. Murakami R, Hirai T, Sugahara T, Fukuoka H, Toya R, Nishimura S, Kitajima M, Okuda T, Nakamura H, Oya N, et al: Grading astrocytic tumors by using apparent diffusion coefficient parameters: Superiority of a one- vs. two-parameter pilot method. Radiology 251: 838-845, 2009.

15. Fan GG, Deng QL, Wu ZH and Guo QY: Usefulness of diffusion/perfusion-weighted MRI in patients with non-enhancing supratentorial brain gliomas: A valuable tool to predict tumour grading? Br J Radiol 79: 652-658, 2006.

16. Kinoshita M, Arita H,Okita Y,Kagawa N, Kishima H, Hashimoto N, Tanaka H, Watanabe Y, Shimosegawa E, Hatazawa J, et al: Comparison of diffusion tensor imaging and (11)C-methionine positron emission tomography for reliable prediction of tumor cell density in gliomas. J Neurosurg 125: 1136-1142, 2016.

17. Beppu T, Inoue T, Shibata Y, Yamada N, Kurose A, Ogasawara K, Ogawa A and Kabasawa H: Fractional anisotropy value by diffusion tensor magnetic resonance imaging as a predictor of cell density and proliferation activity of glioblastomas. Surg Neurol 63: D56-D61, 2005.

18. Ishiwata K, Kubota K, Murakami M, Kubota R and Senda M: A comparative study on protein incorporation of $\mathrm{L}$-[methyl-3H] methionine, L-[1-14C]leucine and L-2-[18F]fluorotyrosine in tumor bearing mice. Nucl Med Biol 20: 895-899, 1993.

19. Jager PL, Vaalburg W, Pruim J, de Vries EG, Langen KJ and Piers DA: Radiolabeled amino acids: Basic aspects and clinical applications in oncology. J Nucl Med 42: 432-445, 2001.

20. Stern PH, Wallace CD and Hoffman RM: Altered methionine metabolism occurs in all members of a set of diverse human tumor cell lines. J Cell Physiol 119: 29-34, 1984.

21. Herholz K, Hölzer T, Bauer B, Schröder R, Voges J, Ernestus RI, Mendoza G, Weber-Luxenburger G, Löttgen J, Thiel A, et al: 11C-methionine PET for differential diagnosis of low-grade gliomas. Neurology 50: 1316-1322, 1998.

22. Pirotte B, Goldman S, Dewitte O, Massager N, Wikler D, Lefranc F, Ben Taib NO, Rorive S, David P, Brotchi J and Levivier M: Integrated positron emission tomography and magnetic resonance imaging-guided resection of brain tumors: A report of 103 consecutive procedures. J Neurosurg 104: 238-253, 2006.

23. Galanis E, Buckner JC, Maurer MJ, Sykora R, Castillo R, Ballman KV and Erickson BJ: Validation of neuroradiologic response assessment in gliomas: Measurement by RECIST, two-dimensional, computer-assisted tumor area and computer-assisted tumor volume methods. Neuro Oncol 8: 156-165, 2006.

24. Gállego Pérez-Larraya J, Lahutte M, Petrirena G, Reyes-Botero G, González-Aguilar A, Houillier C, Guillevin R, Sanson M, Hoang-Xuan K and Delattre JY: Response assessment in recurrent glioblastoma treated with irinotecan-bevacizumab: Comparative analysis of the Macdonald, RECIST, RANO and RECIST + F criteria. Neuro Oncol 14: 667-673, 2012.

25. Shah GD, Kesari S, Xu R, Batchelor TT, O'Neill AM, Hochberg FH, Levy B, Bradshaw J and Wen PY: Comparison of linear and volumetric criteria in assessing tumor response in adult high-grade gliomas. Neuro Oncol 8: 38-46, 2006.

This work is licensed under a Creative Commons Attribution-NonCommercial-NoDerivatives 4.0 International (CC BY-NC-ND 4.0) License. 